\title{
OPTIMAL INPUT DESIGN FOR LOW-DIMENSIONAL SYSTEMS: AN HALDANE KINETICS EXAMPLE
}

\author{
K.J. Keesman* and J.D. Stigter* \\ Systems and Control Group, Wageningen University \\ Mansholtlaan 10, 6708 PA Wageningen \\ THE NETHERLANDS \\ Tel. +31 317 483780, Fax: +31317 484819, \\ e-mail: karel.keesman@wur.nl
}

Keywords: Optimal Input Design, Identification, Singular Optimal Control, Bioreactors.

\begin{abstract}
In this paper the well-known problem of optimal input design is considered. The problem is formulated as follows: given a dynamic non-linear model structure which is assumed to be affine in the input, and a specific parameter of interest $\theta_{k}$, find a feedback law that maximizes the sensitivity of the model output to the parameter. Analytical solutions to this specific problem for a general single state model structure are presented. As an example a bioreactor with a biomass that grows according to the well-known Haldane kinetics under regulated biomass concentration is considered.
\end{abstract}

\section{Introduction}

A natural question in experimental modelling is how should the input sequence be chosen such that the unknown model parameters can be optimally estimated. This is the well known problem of 'optimal' input design (see e.g. Goodwin and Payne (1977); Zarrop (1979); Walter and Pronzato (1990)).

In the late eighties Munack and co-workers (see Munack (1989) and Munack and Posten (1989)) provided a numerical solution to the optimal input design problem for the estimation of the Monod parameters in a bioreactor model using a so-called modified $E$-criterion. Optimal feed rate profiles to provide good estimation conditions for $\mu_{\max }$ and $K_{S}$ have also been obtained in an 'ad hoc' manner using control parametrization (see Versyck (2000)). It has been recognized that numerical solutions to these type of problems are very difficult to find. Recently, Stigter and Keesman (2001) and Keesman and Stigter (2002) have shown how a closely related problem for a fed-batch reactor can be solved analytically using the minimum principle of Pontryagin. In these papers a sequential input design for $\mu_{\max }$ and $K_{S}$ in a bioreactor under different conditions, has been considered.

In this paper the previous results are generalized, but it still focusses on sequentially finding a control law that maximizes the parametric sensitivity $\frac{\partial y}{\partial \theta_{k}}$ for the specific parameter $\theta_{k}$, just to allow more or less simple analytical solutions and thus to avoid the numerical problems frequently mentioned in the cited references! Hence, the classical optimal input design problem is reformulated by decomposing it into a number of related, but simpler, subproblems and thus attacked from a slightly different angle. The goal of the paper is to present these general results and further demonstrate it to the Haldane kinetic case, in particular for the estimation of the Haldane parameters $\mu_{\max }, K_{P}$ and $K_{I}$. This particular choice for Haldane kinetics is motivated as follows: (i) in addition to Monod, Haldane kinetics are widely used, easily understood and under substrate-limiting or inhibition conditions $K_{P}$ and $K_{I}$ are crucial parameters, and (ii) relatively simple solutions to the optimal input design problem appear.

In section 2 first the problem is formulated and the idea of optimal input design via direct parametric sensitivity control is further worked out. In particular, the theory of singular optimal control is used to solve the problem. Then, in section 3 some simulation results are presented and discussed. Finally, the paper finishes with some concluding remarks.

\section{Optimal Input Design}

\subsection{Problem formulation}

Consider the following general state equation affine in $u$ :

$$
\frac{d x}{d t}=f(x, \theta)+g(x, \theta) u
$$

where $u, x \in \mathbb{R}$ and $\theta \in \mathbb{R}^{p}$. The corresponding parametric sensitivity equation with, $x_{\theta} \triangleq \frac{\partial x}{\partial \theta_{k}}$ and $x_{\theta}(0)=0$, becomes:

$$
\frac{d x_{\theta}}{d t}=\left(f_{x}(x, \theta)+u g_{x}(x, \theta)\right) x_{\theta}+\left(f_{\theta}(x, \theta)+u g_{\theta}(x, \theta)\right)
$$


where in what follows the functions and their derivatives are denoted as $f, f_{x}, f_{\theta}$, etc, thus without arguments and without a reference to the parameter index $k$. Furthermore, it is assumed that $f, g \in \mathcal{C}^{3}[(-\infty, \infty) \times(-\infty, \infty)]$. The objective is now to maximize the parametric sensitivity associated with one specific parameter, $\theta_{k}$ from the set $\left\{\theta_{i}, i=1, \ldots, p\right\}$ under the assumption that the state can be directly measured, so that $y=x$ and thus $y_{\theta}=x_{\theta}$. Extension to the case with indirect state measurements i.e

$$
y=h(x, \theta)
$$

so that $y_{\theta}=h_{x} x_{\theta}+h_{\theta}$, is straightforward and will not be treated any further here. Hence, the following quadratic cost function must be maximized,

$$
J=\int_{0}^{t_{f}} x_{\theta}^{2} d \tau
$$

which is directly related to the Fisher information matrix for the scalar case $y=x$. The time $t_{f}$ indicates the final time of the experiment. Let the Hamiltonian $\mathcal{H}$ for the associated optimization problem $(\max \{J\}=\min \{-J\})$ be defined as

$$
\begin{aligned}
\mathcal{H} \triangleq & -x_{\theta}^{2}(t)+\lambda_{1}[f(x, \theta)+g(x, \theta) u \\
& +\lambda_{2}\left[\left(f_{x}+u g_{x}\right) x_{\theta}+\left(f_{\theta}+u g_{\theta}\right)\right]
\end{aligned}
$$

Pontryagin's minimum principle states that the input $u(t) \in \mathbb{U}$, an admissible set of input trajectories, that minimizes $\mathcal{H}$ is optimal and thus in this case maximizes $J$.

In the next section the theory of singular optimal control will be used to obtain a solution to the problem.

\subsection{Singular Optimal Control}

Since the Hamiltonian $\mathcal{H}$ does not explicitly depend on time a first integral of the problem is $\mathcal{H}=$ constant. Also, since the final time $t_{f}$ is assumed unknown and no terminal conditions are specified (determining the value of the co-states at $t_{f}$ ) this constant can be assumed equal to zero. Furthermore, since the problem is affine in the control variable $u(t)$, three possible minima can be found: $u(t)=u_{\max }$ if $s f<0, \quad 0 \leq u(t) \leq u_{\max }$ if $s f=0$ and $u(t)=0$ if $s f>0$, where $s f \triangleq \frac{\partial \mathcal{H}}{\partial u}$ is the so-called switching function. For this optimal input design problem one finds from (5) $s f=\lambda_{1} g+\lambda_{2}\left(g_{\theta}+x_{\theta} g_{x}\right)$. The case $s f=0$ corresponds to a singular arc. A singular control law that minimizes the Hamiltonian $\mathcal{H}$ over all possible input sequences $u(t)$ can be derived by setting (see A. E. Bryson (Jr.) (1999), Keesman and Stigter (2002))

$$
\forall i \in\{0,1,2, \ldots\}: \quad \frac{d^{i}}{d t^{i}} \frac{\partial \mathcal{H}}{\partial u}=0
$$

In this case with one state equation and one specific parameter only two differentiations are needed to determine $u(t)$ explicitly. From the conditions $\mathcal{H}=0$ and $\frac{\partial \mathcal{H}}{\partial u}=0$, i.e. for $i=0$, we obtain

$$
\begin{aligned}
\lambda_{1} & =\frac{x_{\theta}^{2}\left(g_{\theta}+x_{\theta} g_{x}\right)}{-g\left(f_{\theta}+x_{\theta} f_{x}\right)+f\left(g_{\theta}+x_{\theta} g_{x}\right)} \\
\lambda_{2} & =\frac{-g x_{\theta}^{2}}{-g f_{\theta}+f g_{\theta}-x_{\theta}\left(g f_{x}-f g_{x}\right)}
\end{aligned}
$$

Consequently, from the case $i=1$, the singular arc condition (or interior boundary condition) can be derived as

$$
\begin{aligned}
& \frac{x_{\theta}\left[f\left(2 g_{\theta}^{2}+5 x_{\theta} g_{\theta} g_{x}+3 x_{\theta}^{2} g_{x}^{2}\right)+g^{2} x_{\theta}\left(f+x_{\theta} f_{x x}\right)-\right.}{\cdots} \\
& \frac{-g\left(f_{\theta}\left(2 g_{\theta}+3 x_{\theta} g_{x}\right)+x_{\theta}\left(2 g_{\theta} f_{x}+f g_{x \theta}+\right.\right.}{\cdots} \\
& \frac{\left.\left.\left.+x_{\theta}\left(3 f_{x} g_{x}+f g_{x x}\right)\right)\right)\right]}{-g\left(f_{\theta}+x_{\theta} f_{x}\right)+f\left(g_{\theta}+x_{\theta} g_{x}\right)}=0
\end{aligned}
$$

which gives three solutions for $x_{\theta}$ provided $g\left(f_{\theta}+x_{\theta} f_{x}\right)+$ $f\left(g_{\theta}+x_{\theta} g_{x}\right) \neq 0$.

Finally, the case $i=2$, under the interior boundary condition (9), gives the optimal input

$$
\begin{aligned}
& u^{*}(t)=-\left[f ( g _ { \theta } + x _ { \theta } g _ { x } ) \left(2 f_{\theta}\left(g_{\theta}+3 x_{\theta} g_{x}\right)+x_{\theta}\left(4 f g_{x \theta}+\right.\right.\right. \\
& \left.\left.+x_{\theta}\left(3 f_{x} g_{x}+5 f g_{x x}\right)\right)\right)+g^{2} x_{\theta}\left(f_{\theta}\left(2 f_{x \theta}+3 x_{\theta} f_{x x}\right)+x_{\theta}\right. \\
& \left.\left(f f_{x x \theta}+x_{\theta}\left(f_{x} f_{x x}+f f_{x x x}\right)\right)\right)-g\left(2 f_{\theta}^{2}\left(g_{\theta}+3 x_{\theta} g_{x}\right)+\right. \\
& +x_{\theta} f_{\theta}\left(2 g_{\theta} f_{x}+4 f g_{x \theta}+3 x_{\theta}\left(3 f_{x} g_{x}+2 f g_{x x}\right)\right) \\
& +x_{\theta}\left(2 f g_{\theta} f_{x \theta}+f x_{\theta}\left(g_{x} f_{x \theta}+3 f_{x} g_{x \theta}+2 g_{\theta} f_{x x}+f g_{x x}\right.\right. \\
& \left.\left.\left.+x_{\theta}^{2}\left(3 f_{x}^{2} g_{x}+5 f f_{x} g_{x x}+f\left(g_{x} f_{x x}+f g_{x x x}\right)\right)\right)\right)\right] / \\
& {\left[f\left(g_{\theta}+x_{\theta} g_{x}\right)\left(2 g_{\theta}^{2}+6 x_{\theta} g_{\theta} g_{x}+3 x_{\theta}^{2} g_{x}^{2}\right)-g\left(f _ { \theta } \left(2 g_{\theta}^{2}+\right.\right.\right.} \\
& \left.+6 x_{\theta} g_{\theta} g_{x}+3 x_{\theta}^{2} g_{x}^{2}\right)+x_{\theta}\left(2 g_{\theta}^{2} f_{x}+x_{\theta} g_{x}\left(-4 f g_{x \theta}+x_{\theta}\right.\right. \\
& \left.\left.\left.\left(3 f_{x} g_{x}-4 f g_{x x}\right)\right)-2 g_{\theta}\left(f g_{x \theta}+x_{\theta}\left(-3 f_{x} g_{x}+f g_{x x}\right)\right)\right)\right) \\
& +g^{3} x_{\theta}^{2}\left(f_{x x \theta}+x_{\theta} f_{x x x}\right)+g^{2} x_{\theta}\left(-f_{\theta}\left(2 g_{x \theta}+3 x_{\theta} g_{x x}\right)+\right. \\
& +x_{\theta}\left(-g_{x} f_{x \theta}+g_{\theta} f_{x x}-f_{x}\left(3 g_{x \theta}+4 x_{\theta} g_{x x}\right)-\right. \\
& \left.\left.\left.-f g_{x x \theta}-f x_{\theta} g_{x x x}\right)\right)\right]
\end{aligned}
$$

provided that the denominator is unequal to zero.

Notice that after substitution of (9) in (10) a feedback law that is completely determined by the system functions $f$ and $g$ and their derivatives is found. The solution of (9): $x_{\theta}(t)=0$ results in the feedback law $u^{*}(t)=-\frac{f_{\theta}}{g_{\theta}}$. This solution clearly minimizes the cost function (4). In conclusion, Eqn. (10) together with the singular condition (9) determine an optimal sensitivity trajectory in state space. The condition (9) determines when to switch from a 'bang' input to the singular control law (10). We summarize this 
main result in the following theorem for the case of a onedimensional state $x(t)$ (without loss of generality).

Theorem 1 Given the model structure (1) and if $\mathcal{H}\left(x^{*}(t), u^{*}(t), \lambda^{*}(t)\right) \leq \mathcal{H}\left(x^{*}(t), u(t), \lambda^{*}(t)\right)$, the optimal singular control law that maximizes the cost function (4), containing the parametric sensitivity $x_{\theta}$ of $x$ with respect to $\theta_{k}$ (Eqn. 2) for $x_{\theta} \neq 0$ is given by Eqn. (10) under the singular arc condition given by Eqn. (9).

Remark 1 If $g(x, \theta)=b$, a constant input parameter, then the optimal singular feedback law reduces to

$$
\begin{gathered}
u^{*}(t)=g^{2} x_{\theta}\left(f_{\theta}\left(2 f_{x \theta}+3 x_{\theta} f_{x x}\right)+x_{\theta}\right. \\
\left.\left(f f_{x x \theta}+x_{\theta}\left(f_{x} f_{x x}+f f_{x x x}\right)\right)\right) / \\
+g^{3} x_{\theta}^{2}\left(f_{x x \theta}+x_{\theta} f_{x x x}\right)
\end{gathered}
$$

with interior boundary condition: $x_{\theta}=-\frac{f_{x \theta}}{f_{x x}}$. Substitution of this condition in the feedback law gives results as in Keesman and Stigter (2002).

In the next section the theory will be illustrated to an experiment design for the estimation of Haldane parameters in a fed-batch reactor.

\section{Results and Discussion}

\subsection{Fed-Batch Bioreactor Modelling}

Consider a fermentation process with Haldane type growth kinetics. The specific growth rate $\mu\left(C_{S}\right)$ is then given by

$$
\mu\left(C_{S}\right)=\mu_{\max } \frac{C_{S}}{K_{P}+C_{S}+\frac{C_{S}^{2}}{K_{I}}}
$$

where $C_{S}$ is the substrate concentration and $\mu_{\max }, K_{P}$ and $K_{I}$ are specific constants (see Fig. 1).

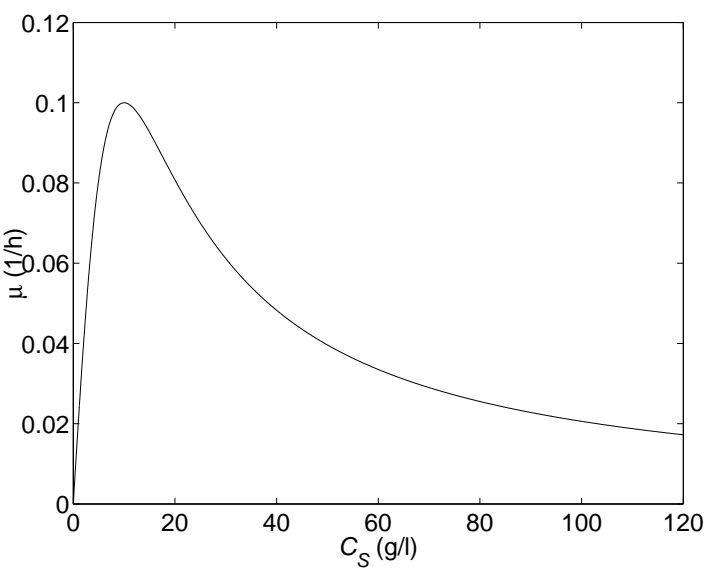

Fig. 1: Haldane kinetics.
Under fed-batch conditions the following mass balance equations holds:

$$
\begin{gathered}
\frac{d C_{S}}{d t}=-\frac{\mu\left(C_{S}\right)}{Y_{X / S}} C_{X}+\frac{F}{V}\left(u-C_{S}\right) \\
\frac{d C_{X}}{d t}=\mu\left(C_{S}\right) C_{X}-\frac{F}{V} C_{X} \\
\frac{d V}{d t}=F
\end{gathered}
$$

where $\mu\left(C_{S}\right)$ is the specific growth rate (see 12$)$ in $1 / \mathrm{h}, 0<$ $Y_{X / S}<1(-)$ the yield coefficient of biomass on substrate, $F(\mathrm{l} / \mathrm{h})$ the flow rate, $V(\mathrm{l})$ the volume of the reactor and $u=C_{S, i n}(\mathrm{~g} / \mathrm{l})$ the variable substrate concentration in the influent. In order to avoid the effect of variations in the biomass concentration on the input design, $C_{X}$ is kept constant at $C_{X}^{*}$, the biomass set-point, by selecting

$$
F=\mu\left(C_{S}\right) V
$$

Hence, the flow rate $F$ is related to the biomass growth and the actual volume of the reactor. Consequently,

$$
\frac{d C_{S}}{d t}=\mu\left(C_{S}\right)\left[-C_{S}-\frac{C_{X}^{*}}{Y_{X / S}}+u\right]
$$

Notice that this dynamic state equation is affine in $u$. As before the optimal input design problem is translated into an optimal parametric sensitivity problem, but let us first investigate the partial sensitivities $\left(f_{x}+u g_{x}\right)$ and $\left(f_{\theta}+u g_{\theta}\right)$ in (2) for the parameters in the Haldane kinetics model. The partial sensitivities are given by

$$
\begin{gathered}
\frac{\partial f+g u}{\partial C_{S}}=-\frac{K_{I} \mu_{\max }\left[K_{I} K_{P} C_{X} *+2 K_{I} K_{P} Y_{X / S} C_{S}+\right.}{\cdots} \\
\frac{\left.\left(K_{I} Y_{X / S}-C_{X}^{*}\right) C_{S}^{2}+Y_{X / S} u\left(C_{S}^{2}-K_{I} K_{P}\right)\right]}{Y_{X / S}\left(K_{I} K_{P}+K_{I} C_{S}+C_{S}^{2}\right)^{2}}
\end{gathered}
$$

$$
\begin{gathered}
\frac{\partial f+g u}{\partial \mu_{\max }}=-\frac{K_{I} C_{S}\left(\frac{C_{X}^{*}}{Y_{X / S}}-u+C_{S}\right)}{\left(K_{I} K_{P}+K_{I} C_{S}+C_{S}^{2}\right)} \\
\frac{\partial f+g u}{\partial K_{P}}=\frac{\mu_{\max } K_{I}^{2} C_{S}\left(\frac{C_{X}^{*}}{Y_{X / S}}-u+C_{S}\right)}{\left(K_{I} K_{P}+K_{I} C_{S}+C_{S}^{2}\right)^{2}} \\
\frac{\partial f+g u}{\partial K_{I}}=-\frac{\mu_{\max } C_{S}^{3}\left(\frac{C_{X}^{*}}{Y_{X / S}}-u+C_{S}\right)}{\left(K_{I} K_{P}+K_{I} C_{S}+C_{S}^{2}\right)}
\end{gathered}
$$

where in what follows e.g. $\frac{\partial C_{S}}{\partial K_{P}}$ with $x=C_{S}$ is simply denoted as $x_{K_{P}}$.

On the basis of these equations, (17)-(21), the corresponding parametric sensitivity equation can be evaluated and an optimal input design for the estimation of a specific parameters can be found. In the next section feedback laws will be derived for optimal input design of the Haldane parameters. 


\subsection{Parametric Sensitivity Control}

Let the fed-batch bioreactor model be given by (17). Hence, $f(x, \theta) \triangleq-\mu\left(C_{S}\right)\left[C_{S}+\frac{C_{X}^{*}}{Y_{X / S}}\right]$ and $g(x, \theta) \triangleq \mu\left(C_{S}\right)$ with $\mu\left(C_{S}\right)$ given by (12). After taking derivatives and substitution in Eqn. (10) an optimal input for the estimation of $\mu_{\max }$ is found, i.e.

$$
u^{*}=\frac{K_{I}^{2} K_{P}^{2} C_{X}^{*}+2 K_{I}^{2} K_{P} C_{X}^{*} C_{S}+K_{I} K_{P}\left(4 C_{X}^{*}+K_{I} Y_{X / S}\right) C_{S}^{2}+}{\cdots} \frac{+4 K_{I} K_{P} Y_{X / S} C_{S}^{3}+\left(K_{I} Y_{X / S}-C_{X}^{*}\right) C_{S}^{4}}{\frac{1}{Y_{X / S}\left(K_{I}^{2} K_{P}^{2}+2 K_{I}^{2} K_{P} C_{S}+4 K_{I} K_{P} C_{S}^{2}-C_{S}^{4}\right)}}
$$

under the interior boundary condition

$$
x_{\mu_{\max }}=-\frac{K_{I} K_{P} C_{S}+K_{I} C_{S}^{2}+C_{S}^{3}}{\mu_{\max }\left(K_{I} K_{P}-C_{S}^{2}\right)}
$$

where $K_{I}^{2} K_{P}^{2}+2 K_{I}^{2} K_{P} C_{S}+4 K_{I} K_{P} C_{S}^{2}-C_{S}^{4} \neq 0$ and $C_{S} \neq \sqrt{K_{I} K_{P}}$. The question is now how to choose the initial substrate concentration $C_{S}(0)$. Recall that $x_{\theta}(0)=$ 0 , i.e the initial parametric sensitivity does not depend on the parameter values. Hence, if one wants to start directly on a singular arc, $C_{S}(0)$ much be chosen such that $K_{I} K_{P} C_{S}+K_{I} C_{S}^{2}+C_{S}^{3}=0$. Choosing $C_{S}(0)=0$ and thus $u^{*}=\frac{C_{X}^{*}}{Y_{X / S}}$ leads to a minimum of $J$ ! However, from (23) it can be easily verified that no real positive initial concentration exists such that the experiment can immediately start on a singular locus. Hence, the input should be of an impulse-bang-singular type. It has been found by a numerical line search that $C_{S}(0) \approx \sqrt{K_{I} K_{P}}$ is a good starting point. However, since in practice the parameters are unknown we start at $C_{S}(0)=4 \mathrm{~g} / \mathrm{l}$ to avoid singularities in the interior condition. A simulated experiment, setting $\mu_{\max }=2.11 / \mathrm{h}, K_{P}=100 \mathrm{~g} / \mathrm{l}, K_{I}=1$ $\mathrm{g} / \mathrm{l}$ and $Y_{X / S}=0.5$, for the estimation of $\mu_{\max }$ is presented in Fig. 2. Notice that first a input pulse is given to the system followed by a bang, where the input concentration is set to zero. The estimates of $\mu_{\max }$ are obtained by using a simple Newton-like observer with gain $\gamma=0.05$.
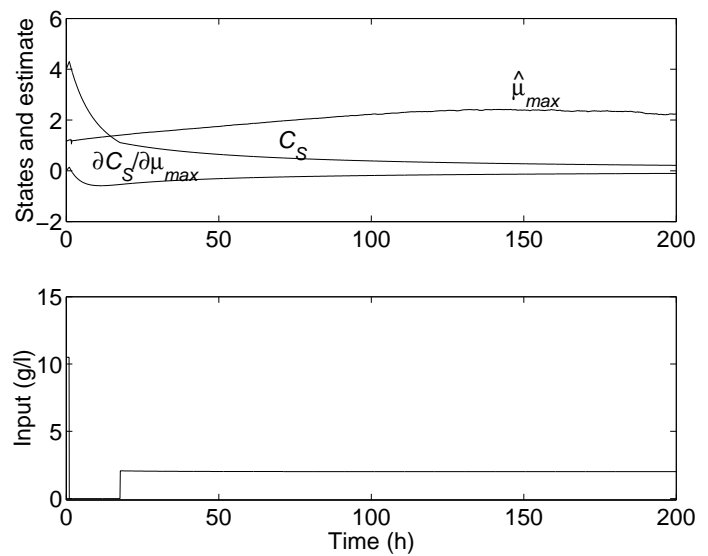

Fig. 2: Optimal experiment for the estimation of $\mu_{\max }$.
The sensitivity of $x_{\mu_{\max }}$ for a deviation in the optimal control input is depicted in Fig. 3.

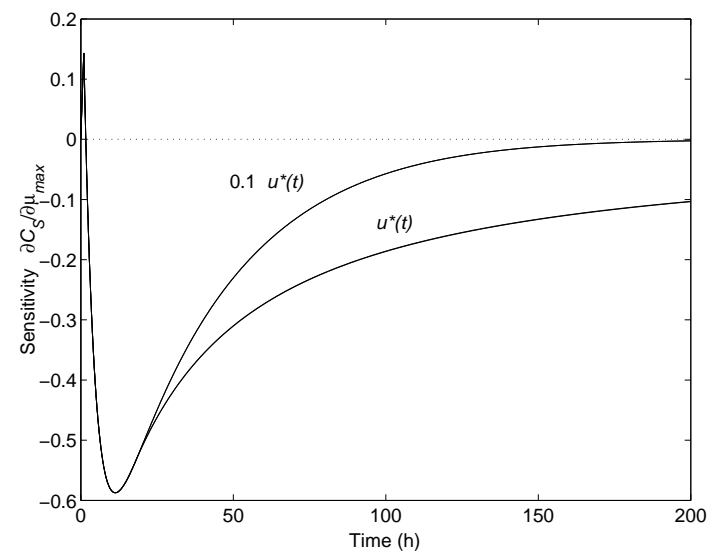

Fig. 3: Effect of deviation on $u^{*}(t)$ on sensitivity.

Similarly, an optimal experiment design can be performed for $K_{P}$, leading to the singular feedback law,

$$
u^{*}=\frac{K_{I} K_{P} C_{X}^{*}+C_{X}^{*} C_{S}^{2}+2 Y_{X / S} C_{S}^{3}}{Y_{X / S}\left(K_{I} K_{P}+C_{S}^{2}\right)}
$$

under the interior boundary condition

$$
x_{K_{P}}=\frac{K_{I} C_{S}}{\left(K_{I} K_{P}-C_{S}^{2}\right)}
$$

and assuming that $C_{S} \neq \sqrt{K_{I} K_{P}}$. It appears again that $C_{S}(0) \approx \sqrt{K_{I} K_{P}}$ is a good starting point. The results of the simulated experiment, using the same parameter values as before but now setting $\gamma=0.01$, for optimal estimation of $K_{P}$ are presented in Fig. 4. Notice that due to the total removal of the substrate the experiment is limited to $100 \mathrm{~h}$.
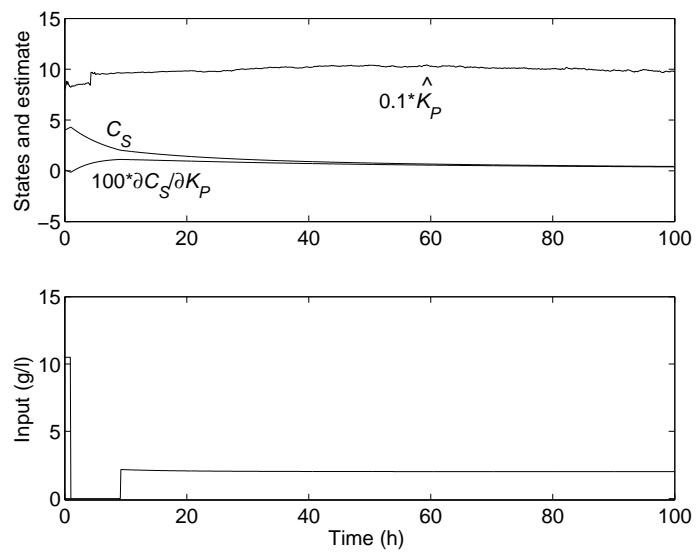

Fig. 4: Optimal experiment for the estimation of $K_{P}$. 
Finally, for the parameter $K_{I}$ it has been found that

$$
u^{*}=\frac{-3 K_{I} K_{P} C_{X}^{*}-2 K_{I} K_{P} Y_{X / S} C_{S}+C_{X}^{*} C_{S}^{2}}{Y_{X / S}\left(-3 K_{I} K_{P}+C_{S}^{2}\right)}
$$

under the interior boundary condition

$$
x_{K_{I}}=\frac{C_{S}^{3}}{K_{I}\left(-K_{I} K_{P}+C_{S}^{2}\right)}
$$

and assuming that $C_{S} \neq \sqrt{3 K_{I} K_{P}}$ and $C_{S} \neq \sqrt{K_{I} K_{P}}$. Unlike the previous cases it now appears that a high initial substrate concentration $C_{S}(0)$ is a good starting point. The results of the simulated experiment, using the same parameter values as before but changing the initial substrate concentration to $C_{S}(0)=8 \mathrm{~g} / 1$ and $\gamma=0.01$, for optimal estimation of $K_{I}$ are presented in Fig. 5 and 6.
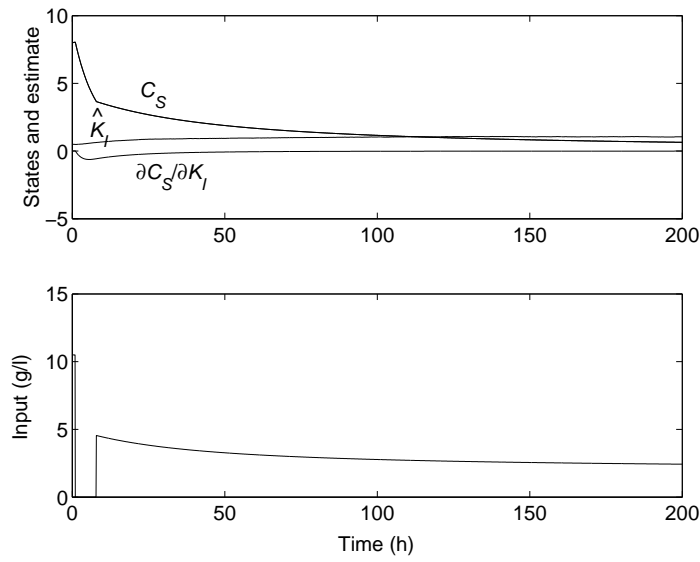

Fig. 5: Optimal experiment for the estimation of $K_{I}$.
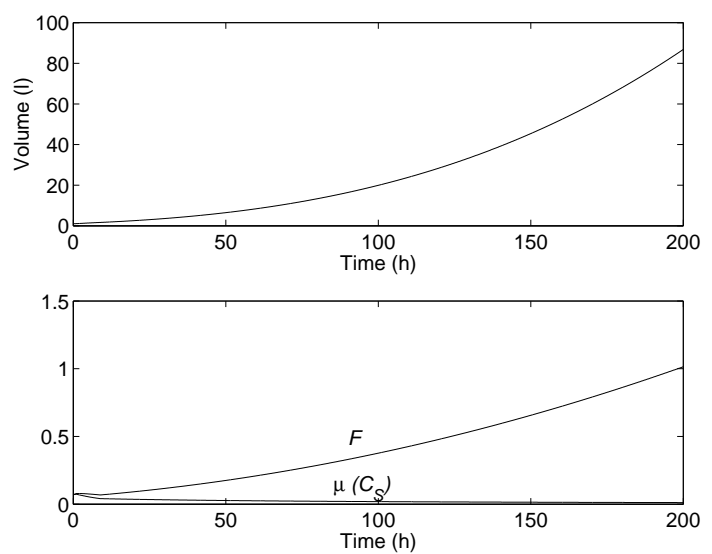

Fig. 6: Hydraulic behaviour reactor.

Notice that instead of solving the full optimal design problem for all parameters together, we propose here to solve it sequentially. Clearly, solving the full example problem leads to four states (i.e. substrate concentration and three parametric sensitivities) with four co-state equations. Hence, in order to obtain a control law that only depends on the states four co-states have to be eliminated. In general this problem is too complex to solve it analytically. Therefore the sequential procedure, decomposing the problem into smaller problems for which feedback laws can be rather easily found using symbolic software, has been proposed. Obviously, in each separate experiment full attention is given to the reduction of the estimation uncertainty of a specific parameter, which will most often also affect the uncertainty in the other parameters. Because in general the prior knowledge of parameters values is limited an iterative procedure is suggested. The sensitivity of $J=\int_{0}^{t_{f}} x_{\theta}^{2} d \tau$ with $t_{f}=100 \mathrm{~h}$ to normalized errors in the parameters $( \pm 10 \%)$ using a factorial design for the deviations is summarized in Table 1 . The center point is defined by: $\left[\begin{array}{lll}\bar{\mu}_{\max } & \bar{K}_{P} & \bar{K}_{I}\end{array}\right] \triangleq\left[\begin{array}{lll}2.1 & 100 & 1\end{array}\right]$ where the overbar denotes the nominal value. In the last row of this Table the normalized center point is indicated by zeros. In this sensitivity study the same initial conditions as in the simulation study are used.

Table 2 contains the parameter estimates $\hat{\beta}_{0}, \ldots, \hat{\beta}_{3}$, i.e the parameter sensitivities and associated standard deviations of $J$ to errors in the kinetic model parameters, of the linear regression model,

$$
J=\beta_{0}+\beta_{1} X_{1}+\beta_{2} X_{2}+\beta_{3} X_{3}+e
$$

where $X_{1}=\frac{\mu_{\max }-\bar{\mu}_{\max }}{0.1 \bar{\mu}_{\max }}, X_{2}=\frac{K_{P}-\bar{K}_{P}}{0.1 \bar{K}_{P}}, X_{3}=\frac{K_{I}-\bar{K}_{I}}{0.1 \bar{K}_{I}}$. Clearly for $10 \%$ deviation there holds: $X_{i}= \pm 1$ for $i=$ $1, \ldots, 3$ (see Table 1 ). It appears that the error $e$ for each of the three experiments is smaller than $0.03,2.10^{-5}$ and 0.22 , respectively. Hence the residuals are relatively small. Furthermore, for the optimal experiment design for $\mu_{\max }$ it appears that the $10 \%$ errors in parameter $K_{I}$ does not affect the cost $J$ and the cost in the experiment for $K_{I}$ is not affected by errors in $\mu_{\max }$. In addition to this, using an overall curvature check, it turned out that second-order terms in this regression-based sensitivity analysis do play a minor role and can thus be neglected.

\subsection{Concluding remarks}

In this paper, for a general one-dimensional model structure affine in $u$, a feedback law for optimal input design of a specific parameter has been derived. Implementation of this feedback law leads to the maximization of a quadratic cost function containing the associated parametric sensitivity.

The approach is illustrated to the dynamic input design for the estimation of Haldane kinetic parameters in a fedbatch bioreactor. In the experimental set-up the biomass concentration is kept constant by manipulating the flow, so that effectively only one state equation remains. 
Table 1: Factorial design parameter perturbations.

\begin{tabular}{ccc|c|ccc|c|ccc|c}
\hline \multicolumn{1}{l}{$\partial C_{S} / \partial \mu_{\max }$} & \multicolumn{1}{c}{$\partial C_{S} / \partial K_{P}$} & $* 10^{-4}$ & \multicolumn{3}{l}{$\partial C_{S} / \partial K_{I}$} \\
\hline- & - & - & 2.11 & - & - & - & 6.94 & - & - & - & 4.28 \\
\hline+ & - & - & 1.55 & + & - & - & 7.73 & + & - & - & 4.13 \\
\hline- & + & - & 1.80 & - & + & - & 4.12 & - & + & - & 3.15 \\
\hline+ & + & - & 1.35 & + & + & - & 4.67 & + & + & - & 3.10 \\
\hline- & - & + & 2.11 & - & - & + & 7.23 & - & - & + & 2.07 \\
\hline+ & - & + & 1.55 & + & - & + & 8.03 & + & - & + & 1.98 \\
\hline- & + & + & 1.81 & - & + & + & 4.29 & - & + & + & 1.52 \\
\hline+ & + & + & 1.36 & + & + & + & 4.84 & + & + & + & 1.48 \\
\hline 0 & 0 & 0 & 1.67 & 0 & 0 & 0 & 5.76 & 0 & 0 & 0 & 2.47 \\
\hline
\end{tabular}

Table 2: Parameter sensitivities.

\begin{tabular}{c|c|c|c}
\hline & $\partial C_{S} / \partial \mu_{\max }$ & $\partial C_{S} / \partial K_{P} * 10^{-4}$ & $\partial C_{S} / \partial K_{I}$ \\
\hline$\beta_{0}$ & $1.701 \pm 0.012$ & $5.960 \pm 0.043$ & $2.687 \pm 0.069$ \\
\hline$\beta_{1}$ & $-0.251 \pm 0.013$ & $0.336 \pm 0.045$ & $-0.042 \pm 0.073$ \\
\hline$\beta_{2}$ & $-0.126 \pm 0.013$ & $-1.501 \pm 0.045$ & $-0.399 \pm 0.073$ \\
\hline$\beta_{3}$ & $0.002 \pm 0.013$ & $0.116 \pm 0.045$ & $-0.950 \pm 0.073$ \\
\hline
\end{tabular}

\section{References}

A. E. Bryson (Jr.) (1999). Dynamic Optimization. Addison Wesley.

Goodwin, G. and Payne, R. (1977). Dynamic System Identification: Experiment Design and Data Analysis. Prentice-Hall, Englewood Cliffs.

Keesman, K. J. and Stigter, J. D. (2002). Optimal Parametric Sensitivity Control for the Estimation of Kinetic parameters in Bioreactors. Math. BioSc., 179:95-111.

Munack, A. (1989). Optimal feeding strategy for identification of monod-type models by fed-batch experiments. In N.M. Fish et al. (Eds.), Computer applications in fermentation technology, modelling and control of biotechnological processes, Elsevier, Amsterdam, pages 195-204.

Munack, A. and Posten, C. (1989). Design of optimal dynamical experiments for parameter estimation. In Proc. American Control Conference 1989, pages 20102016.

Stigter, J. D. and Keesman, K. J. (2001). Optimal parametric sensitivity control for a fed batch reactor. In Proceedings European Control Conference 2001, pages 2841-2844. Porto, Portugal, 4-7 Sep.

Versyck, K. J. (2000). Dynamic Input Design for Optimal Estimation of Kinetic Parameters in Bioprocess Models. $\mathrm{PhD}$ thesis, Katholieke Universiteit Leuven.
Walter, E. and Pronzato, L. (1990). Qualitative and quantitative experiment design for phenomenlogical models - a survey. Automatica, 26(2):195-213.

Zarrop, M. (1979). Optimal Experiment Design for Dynamic System Identification. Springer-Verlag, Heidelberg. 